\title{
Multipurpose Layout Planner
}

\author{
1A. Gomes de Alvarenga, F.J. Negreiros-Gomes, Hannu Ahonen+ \\ 2H. J. Pinheiro-Pita , L. M. Camarinha-Matos \\ 1Universidade Federal do Espírito Santo \\ Av. Fernando Ferrari S/N - CEP 29060-900, Vitória- ES, Brasil \\ E-mail: [gomes,negreiro,hannu] @ufes.br \\ 2Universidade Nova de Lisboa \\ Quinta da Torre, 2825 Monte Caparica, Portugal \\ E-mail:[hp,cam]@uninova.pt
}

\begin{abstract}
There are several kinds of layout problems, most of them concerning the allocation of a set of planar objects over a delimited planar area. There are several forms of representation of such problems, depending on their application. In order to treat these layout problems in a unified way, we propose an object-oriented approach that decomposes the problems in generic entities needed by a problem processing engine, the Layout Planner, to form a problem solver instance. This paper concerns the description of a tool for development of decision support systems for layout planning, where the entities are mapped into objects that own a dynamic behaviour. The user describes his/her problem instance in a graphical way using visualisation forms of the objects. A fertile application field of such DSS is in CIM activities such as machine layout and cutting. First, we identify models and approaches for layout problems and present an architecture for the DSS generator and then discuss a case study of the leather cutting problem.
\end{abstract}

Keywords

Layout Optimization, Interactive Planning, CIM systems, Knowledge-Based Systems, Problem Visualisation.

\section{INTRODUCTION}

Industrial cutting and layout problems are N P-complete and are characterised by several specific constraints. As such, exact solution approaches for them are not usually suitable. On the other hand, heuristical approaches can incorporate knowledge in the form of preferences and constraints guiding the search process.

The Multipurpose Layout Planner proposed in this study is based on the observation that in spite of their different contexts, these problems have several common features. Thus the proposed system is not restricted to factory floor layout problems as the expert system presented in [4]. Another feature of the proposed system is the generalised concept of algorithm: instead of an algorithm simply based on the optimization approach, techniques belonging to problem solving and search approaches are also considered. In fact, proposed system includes a catalog of several algorithms to be selected according to the specificities of

+ On leave from VTT Information Technology, Espoo, Finland 
each application scenario. In terms of implementation, the definition of the system follows the object-oriented paradigm, which makes it possible to implement a flexible and extendible problem solving system.

Concerning the level of automation of such system, it can be viewed as a computer aid tool to help an expert planner to design the desired layout, monitoring her/his actions with the objective to provide real time consistency (reactive behaviour) of the design and to improve her/his performance. For example, in the case of leather cutting, there are several handly operations, e.g. pieces' allocation, that obey unstructured rules that can be built in the system. These rules are the representation of the layout problem knowledge that, together with the problem visualisation form, the user supplied parameters and a subset of the algorithm catalogue, identify a problem solver instance.

It shall be noted that the intended user of such system is not necessarily an expert in optimization and problem solving areas. However, he is supposed to be an expert in the application domain, i.e., able to specify constraints of the problem domain, optimization criteria and to evaluate solutions. Therefore, a decision support system is devised to help this user in "configuring" the layout planner for his particular needs.

The paper is organised as follows. In the section 2, we analyse some classes of layout problems concerning the representation, and the approaches for their solution. Section 3 deals with the architecture of the layout planner presenting a description of its functionality. In section 4 , we describe, as application case study, the leather cutting problem.

\section{CLASSES OF LAYOUT PROBLEMS}

The purpose of this work is to develop an integrated tool for decision support in the layout context. In this section, we identify some problem instances that in some way can be characterised as problems of this context.

\subsection{Nesting for Two Dimensional Shapes}

A problem of theoretical importance as well as of industrial interest is that of optimization of two dimensional layout. In this problem, we are concerned with the allocation of a specified number of two dimensional regular or irregular (non-rectangular) pieces on a finite dimensional stock plate, the objective being to minimise the amount of produced waste.

This problem appears in the context of several production processes like steel, clothing manufacturing, wood, and shipbuilding. An interesting application is found in the shoe manufacturing industry, where moulds are cut from leather plates. The problem here belongs to the more generic class of cutting and packing problems in that the leather plates tend to be highly irregular in shape and to contain defective non usable areas.

We shall use a state graph to represent the problem although other approaches can be used. We will make the following assumptions about the problem

- Geometric shapes of the pieces (or leather plate) are approximated by polygons.

- Overlap of pieces is not accepted.

- Rotations of the pieces are allowed only within a bounded range.

We are thus using a representation by a state graph $[1,5]$ where each piece $\mathrm{j}$ as well as the stock plates are stored into the global database represented as polygonal regions with vertices $\left(x_{i, j}, y_{i, j}\right)$

A generic state of the system is characterised by storing into the database all the information relative to a specific layout pattern as illustrated in Figure 1. 


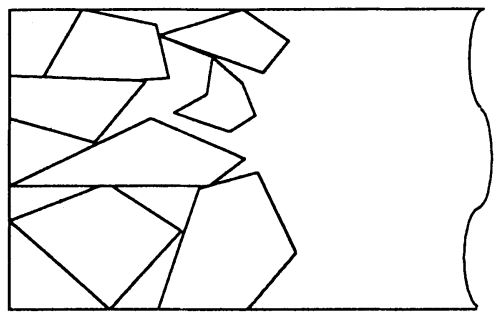

Figure 1 A Generic State of the System.

In this state graph, the production rules are defined by considering the strategies inside the universe of feasible solutions that are applicable to a current state $E_{k}$ allowing the generation of a new state $E_{k}+1$ (a child state of $E_{k}$ ), by the nesting of a new piece to the layout corresponding to the state $E_{k}$

An algorithm to solve the nesting problem based on this approach must take into consideration the solution paths defined from the root of the graph to a generic state $E_{k}$. In this way we can associate to a generic state $E_{k}$ a labelled element $E(k, c, p)$ where $k$ is the terminal state on the path from the root, $c$ is the production cost of the layout associated with $E_{k}$, and $p$ is a pointer to another labelled element $\mathrm{E}(\mathrm{r}, \mathrm{v}, \mathrm{q})$.

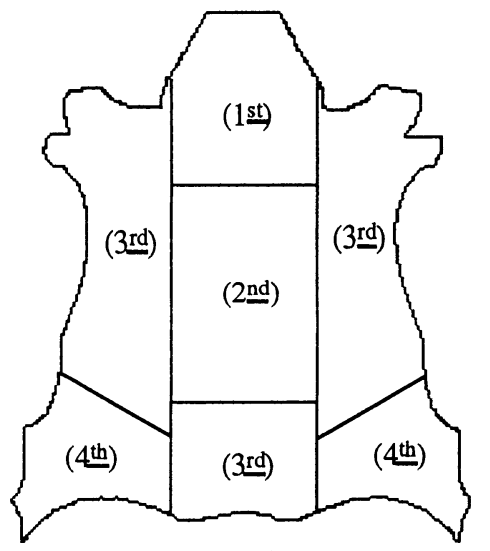

Figure 2 Classification of a leather plate.

Now, related to the nesting of leather plates, it is possible to generate a partition (e.g., the regions 1, 2, 3 and 4 in Figure 2) on the leather plates based on quality classification of the raw material.

We can apply the above approach to each sub-region of the partition in order to obtain a representation of the problem in the form of a state graph.

\subsection{Machine layout problem on a factory floor}

One problem encountered in the design of automated manufacturing system is the layout of machines on a factory floor. This problem can be seen as an application of the facility layout problem in automated manufacturing system [6]. The facility layout problem involves the arrangement of a given number of facilities so that the total cost required by the material handling systems to transport material among the facilities is minimised. One of the most 
frequently used formulations to model the facility layout problem is the quadratic assignment problem, an optimization technique which is concerned with the allocation of discrete entities to discrete locations. This problem is JVP-complete [8]. Considering the computational complexity of the facility layout problem, many heuristic methods have been developed. Kusiak and Heragu [7] classified these as

- construction methods;

- improvement methods;

- hybrid methods;

- graph theoretic methods.

We observe that the basic types of machine layout problems can be represented by a graph search like in the nesting problem. In this graph the root node represents an empty floor.

The rules identify how to assign a new machine to a partial layout. So, effective search process can be used to solve the problem.

\section{THE GENERAL ARCHITECTURE AND FUNCTIONAL DECOMPOSITION}

\subsection{General Layout Planner Overview}

A layout problem can be defined as follows: Given a set of planar objects $O=\left\{o_{1}, o_{2}, \ldots, o_{k}\right\}$ where each object $o_{i}$ has a geometry defined by $G_{o_{i}}=\left\{\left(x_{1}, y_{1}\right), \ldots,\left(x_{n}, y_{n}\right)\right\}$, a planar area $A$ with geometry represented by $\left.G_{A}=\left\{x_{1}^{a}, y_{1}^{a}\right), \ldots,\left(x_{m}^{a}, y_{m}^{a}\right)\right\}$ where $\left(x_{\bullet}, y_{\bullet}\right)$ is xy-axis coordinates, and a set of constraints $R: O \rightarrow A$ representing the objects' behaviour, the problem is to place the object $o_{i}, i=1, \ldots, k$ on $A$, subject to $R$, with the objective of optimising some functional $f(O, A)$.

Depending on the problem's domain as well as the objective function, there are many algorithms to solve that problem. Our approach is to investigate a kind of interactive system that, instead of doing an automatic planning, cooperates with a human expert to achieve an optimised solution for a layout problem.

\subsection{The general architecture of the Layout Planner}

The general architecture of the Layout Planner system is depicted in Figure 3. The system consists of five functional modules: the Taxonomy of Problem's models, the Algorithms Catalog, the Archive of Old Solutions, the User Interface Module, and the Layout Planner Module.

The Taxonomy of Problem's models organises the different classes of problems known by the system. Each problem's model is defined by a Specific Domain Knowledge and a Specific Planning Knowledge. The Domain Knowledge specifies the characteristics of the problem (e.g. application domain, shapes of parts to be allocated, etc.), while the Planning Knowledge, composed by sets of rule bases, specifies the constraints and some rules that should be observed for that problem.

The Algorithm Catalog is a library of algorithm prototypes each of which contains an executable code and an indication about the applicability of the algorithm (meta knowledge) to a problem (model). This module is designed to support maintenance, graphical browsing and explanation facilities about the algorithms. 


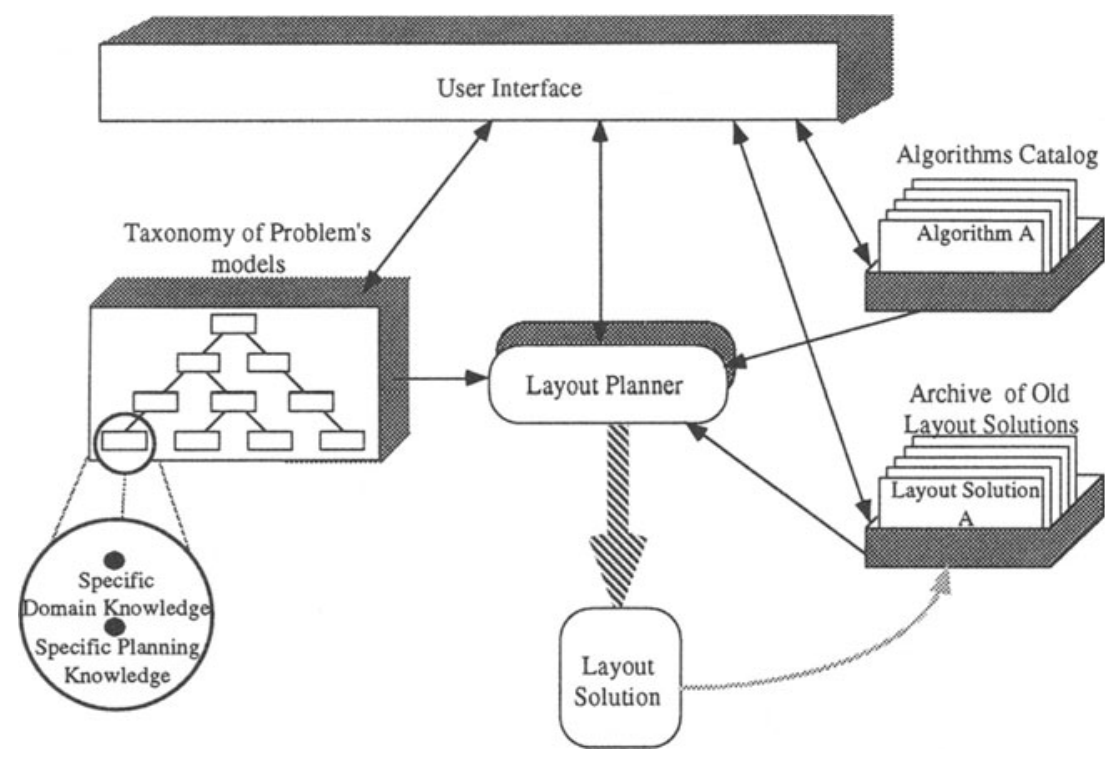

Figure 3: General architecture of the Layout Planner

The Archive of Old Solutions is composed by two archive subsystems, Archive of Problem Instances and Archive of Layout Solutions that allow the user to restart working with problems and solutions constructed before.

The User Interface Module is responsible for the management of the interaction mechanisms. As a platform to support interaction, a set of appropriate interaction forms was developed. An interaction form is a general script that characterises a graphical presentation and an interaction behaviour. In this module, each application object has a different behaviour and appearance depending on the user that interacts with it [3]. The concept of objects with dynamic behavior developed in the CIM-CASE system [9] is applied to this context.

The Layout Planner Module, described in more detailed below, is the central component of the system.

\subsection{The Layout Planning Methodology}

The proposed layout planning methodology is decomposed into four phases: Specification; Selection of Optimization Algorithms, Generation of Layout Solutions, and Selection of a Final Solution(figure 4).

The first phase is started with the selection of a problem's model. As an answer to this action, the Specific Domain and Planning Knowledge are instanciated and, a subset of old solutions, belonging to this model, are selected from the Archive of Old Solutions. The user can choose one of these problem's instances as the starting point or he can start a new specification. In the second case he defines the area where the shapes will be allocated and builds a draft layout, allocating the shapes to that area. Depending on the complexity of the problem, this procedure may need a hierarchical support (e. g. using the shop floor layout problem, three levels can be foreseen: shop level, cell level and component level).

As the construction of the draft layout is an activity requiring various iterations that couldn't be guided by the system, a great flexibility at the interaction mechanisms is needed and the system 
should also provide a set of critics that, based on the Specific Planning Knowledge, validate the user options and make suggestions. For some user actions these critics may be activated automatically (e.g. using the leather cutting problem, if a mould is allocated in the appropriate part of a leather plate) - embedded criticism. For other actions on-demand criticism is more appropriate, i.e., the criticism can only be activated when the user thinks he has achieved a solution (e.g. using the shop floor layout problem and supposing that the user has allocated an assembly robot, if the assembly position was defined).

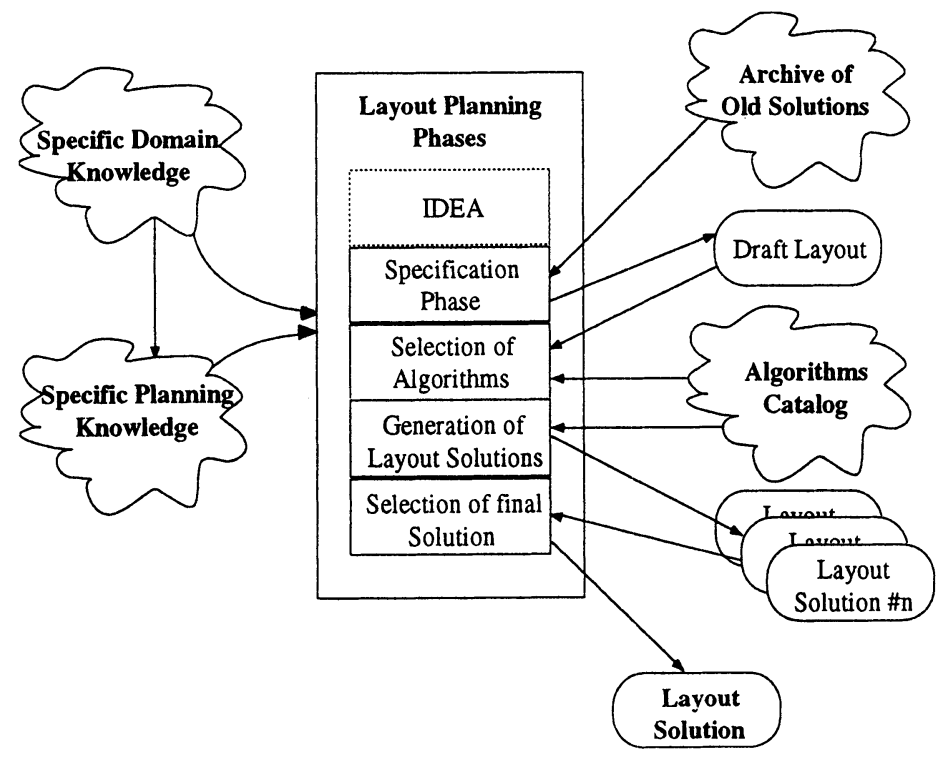

Figure 4: General model of the Layout Planner

In the phase of Selection of Optimization Algorithms, the user specifies a set of optimization criteria. On the basis of this set and taking into account the type o problem to be solved, a set of available algorithms is shown, regarding the appropriate Catalog. The user selects one, or more, of those algorithms and the system generates the formulation of the problem within the selected approaches (e.g. optimization methods or heuristical search methods). This formulation may consist of a mathematical model of the problem with an objective function and constraint equations (optimization methods) or it is given with the help of the definition of search states (heuristical search methods).

In the Generation of the Layout Solutions phase, for each selected algorithm a concrete instance of the processor able to solve the given problem using that algorithm is built. The parameters required to accomplish the computation task are initialised and, sometimes, additional features, like the estimation of path costs for the $\mathrm{A}^{*}$ algorithm, are added. After that, the processors are activated under the control of a Processor Supervisor. This module transmits, to the User Interface, information on the computation status and enables the user to decide if a given processor should be stopped or not (e.g. if it is going to take too much time). On the other hand, the Processor Supervisor saves a trace of the computation of each processor activity to be exploited later.

Finally, in the Selection of a Final Solution phase, the quality of the achieved solutions is evaluated, taking into account the Specific Planning Knowledge, and the best solution is 
selected. This phase sends to the user interface an explanation about the chosen solution, i.e., how the solution was achieved and which alternatives solutions could be available (if any).

At the end, it should be noted that the user is the responsible to accept or refuse the final solution. In the second case he can return to the Selection of Optimization Algorithms and selected a different set of criteria and/or algorithms and repeat the process. In any case, the draft layout is saved in the Archive of Old Solutions (Problem Instances Archive), while the achieved solution will be saved in the Layout Solutions Archive, if and only if it was accepted by the user.

\section{CASE STUDY: LEATHER CUTTING PROBLEM}

Lets now describe the classes of objects of the layout design for the leather cutting problem [3]. The leather plate is represented by the area $\mathrm{A}$ (see section 3.1), which is a two dimensional bounded region. This area has two derived classes: Graded Leather Plate (GLP) and Defective Leather Plate (DLP). An object of the GLP subclass contains additional attributes such as texture, thickness and boundary type. It also incorporates a partition that can be applied to generate, based on a raw material quality criterion, new objects of these subclasses.

DLP object contains a sub region of the leather plate that encloses a defect such as a stain, hole or stretch. A defect operator may be defined mapping the leather plate class into objects of this derived class.

The objects to be placed on the area A (see section 3.1) represent the moulds, which are abstractions of two dimensional regions with attributes that identify the pieces that will be produced by the layout planner, the quality of the required raw material and directional properties.

The classes of leather plate and mould present a set of constraints that represents the interaction of the objects of those classes. Some constraints are as follows:

Overlap: its purpose is to avoid an overlap among the moulds in the layout plan generated by the system over a leather plate.

Direction: Its purpose is to define the set of feasible directions for realising the layout of the leather plate.

Identification: These constraints represent technical specifications of the moulds concerning the leather plate. For example, there are constraints that specify whether a mould may be manufactured or not from a graded leather plate.

\section{CONCLUSIONS}

An architecture for a Multipurpose Layout Planner (MLP) was proposed. This system includes a catalog of optimization algorithms that can be configured to operate in different contexts. A decision support system helps the user in configuring the system for his particular application domain. Examples of applications on the leather cutting and factory layout planning have been used to test the validity of the approach. Due to the dynamic nature of leather cutting, the MLP seems to be a well-fit tool to improve the cutting process performance.

With the purpose to spread the application range of the MLP, we are currently studying the problem of the multilayer shopfloor layout.

\section{ACKNOWLEDGEMENTS}

The authors thank the European Commission in reference to the CIMIS.net project.

The Brazilian authors also thank CNPq (Brazilian Council of Research and Development) which partially supports this research, and the Portuguese authors would like to thank JNICT for supporting the projects where these ideas were developed. 


\section{REFERENCES}

[1] Alvarenga, A.G.; Gomes, F.J.N, Provedel, A.; Sastron, F.; Arnalte, S. (1994) Integration of an Irregular Cutting System into CIM. Part I - Information Flows - Studies in Informatics and Control, Vol. 3. Nos. 2-3, pp. 157-163.

[2] Bell, P.C. (1991) Visual Interactive Modelling: the past, the present and the prospects European Journal of Operational Research, 54, pp. 274-286.

[3] Gomes, F.J.N; Alvarenga, A.G.; Lorenzoni, L.L.; Pinheiro-Pita, H.J.; CamarinhaMatos, L.M.(1994) Objects Dynamic Behaviour for Graphical Interfaces in VIM = An Application Case Study $=$, Studies in Informatics and Control, Vol. 3. Nos. 2-3, pp. 165-171.

[4] Heragu, S.S.; Kusiak, A. (1990) Machine Layout: an Optimization and Knowledge-based approach - Int. Journal Production Research 28, pp. 615-635 .

[5] Nilsson, N. J. - Principles of Artificial Intelligence - Tíoga Publishing Company, Palo Alto, Califórnia.

[6] Kusiak, A. - Intelligent Manufacturing Systems - Prentice-Hall , New York.

[7] Kusiak, A.; Heragu, S.S. (1987) The Facility Layout Problem. European Journal of Operational Research 29, pp. 229-251.

[8] Sahni, S.; Gonzalez, T. (1976) P-Complete Approximaton Problem - J. Assoc. Comput. 23,pp.555-565.

[9]Pinheiro-Pita, H., Camarinha-Matos, L. (1993). Comportamento de Objectos Activos na Interface Gráfica do Sistema CIM-CASE. In $4^{a}$ s. Jomadas Nacionais de Projecto. Planeamento e Produção assistidos por Computador, 1 (pp. 189-198). Lisboa: Ordem dos Engenheiros. [10]Camarinha-Matos, L., 1993 IEEE International Conference on Robotics and Automation, 3 (pp. 63-70). Atlanta, Georgia, EUA: IEEE Computer Society Press.

\section{BIOGRAPHY}

Arlindo Gomes de Alvarenga and Francisco José Negreiros Gomes are Associate Professors at Federal University of Espirito Santo. They received D.Sc. degrees in Systems Engineering and Computer Science from Federal University of Rio de Janeiro in 1982 and 1988, respectively.

Their research interests are in Combinatorial Optimization, Automated Systems for Manufacturing and Decision Support Systems.

Hannu Ahonen is Visiting Professor at Federal University of Espirito Santo, being on leave from VTT-Information Systems, Finland. He received a D.Tech. degree in Mathematics from Helsinki University of Technology in 1982. His research interest is in Combinatorial Optimization and Search Methods of Artificial Intelligence.

Mr Helder J. Pinheiro-Pita is adjunct professor in Instituto Superior de Engenharia de Lisboa. He received his B.Sc. degree by ISEL and his Lic. Eng. degree (five years Portuguese degree) by New University of Lisbon. Now he is finishing his PhD thesis on Interactive Planning applied to the generation of Control Architectures to CIM.

Dr. Luis M. Camarinha-Matos received his Computer Engineering degree and $\mathrm{PhD}$ on Computer Science, topic Robotics and CIM, from the New University of Lisbon. Currently he is auxiliar professor (eq. associate professor) at the Electrical Engineering Department of the New University of Lisbon and leads the group of Robotic Systems and CIM of the Uninova's Center for Intelligent Robotics. His main research interests are: CIM systems integration, Intelligent Manufacturing Systems, Machine Learning in Robotics. 